\title{
Low Prevalence of Antibiotic Resistance and High Level of Antibiotic Consumption in Rural China: Interdisciplinary Study
}

\section{Debin Wang}

Anhui Medical University

Xinrong Shen

Anhui Medical University

Jing Chai

Anhui Medical University

Jing Cheng

Anhui Medical University

Rui Feng

Anhui Medical University

Meixuan Chen

Durham University

Christie Cabral

University of Bristol

Isabel Oliver

Public Health England

Jilu Shen

Fourth Affiliated Hospital of Anhui Medical University

Alasdair MacGowan

North Bristol NHS Trust

Karen Bowker

North Bristol NHS Trust

Matthew Hickman

Bristol Medical School: University of Bristol Medical School

Paul Kadetz

University of Global Health Equity

Linhai Zhao

Anhui Medical University

Yaping Pan

First Affiliated Hospital of Anhui Medical University

Rachel Kwiatkowska

Public Health England

Xiaowen $\mathrm{Hu}$

Anhui Provincial Hospital

Helen Lambert ( $\sim$ h.lambert@bristol.ac.uk)

University of Bristol https://orcid.org/0000-0003-2506-6316

\section{Research}

Keywords: Antibiotic resistance, antibiotic use, China, rural, determinants, respiratory tract infections, mixed methods

Posted Date: March 2nd, 2021

DOI: https://doi.org/10.21203/rs.3.rs-249016/v1

License: (c) (1) This work is licensed under a Creative Commons Attribution 4.0 International License. Read Full License 


\section{Abstract}

Background: We tested the feasibility of determining the prevalence and epidemiology of antibiotic resistance in rural China and investigated patterns and drivers of antibiotic use for common respiratory and urinary tract infections (RTI/UTI).

Methods: (i) Observations and exit interviews in eight village clinics and township health centres and 15 retail pharmacies; (ii) Urine, throat swab and sputum samples from patients to identify potential pathogens and test susceptibility; (iii) 103 semi-structured interviews with doctors, patients, pharmacy workers and antibiotic-purchasing customers; (iv) Assessment of completeness and accuracy of electronic patient records through comparison with observational data.

Results: $87.9 \%$ of 1123 recruited patients were prescribed antibiotics, most of whom had RTIs. Antibiotic prescribing for RTIs was not associated with presence of bacterial pathogens but with longer duration of infection $(\mathrm{OR}=3.33)$ and presence of sore throat (OR=1.64). Fever strongly predicted prescription of intravenous antibiotics ( $\mathrm{OR}=2.87$ ). Resistance rates in bacterial pathogens isolated were low compared with national data. $25.8 \%$ of patients reported antibiotics use pre-clinic visit. Only $56.2 \%$ of clinic patients and $53 \%$ of pharmacy customers could confirm their prescription included antibiotics. Diagnostic uncertainty, economic need, understanding of antibiotics as anti-inflammatory and limited doctor-patient communication were identified as drivers of antibiotic use. Completion and accuracy of e-records was variable.

Conclusions: Despite high levels of antibiotic prescribing and self-medication, prevalence of antibiotic resistance in this rural population is currently relatively low. More systematic use of e-records could improve antibiotic surveillance in rural facilities.

\section{Background}

Antibiotic resistance (ABR) is recognised as a major global health concern, partially driven by non-essential use of antibiotics in medical treatment.(1) China is one of the world's largest consumers of antibiotics, with prescription rates twice that recommended by the World Health Organisation,(2) and there is substantial evidence that antibiotics are overused in healthcare. $(3,4)$ Rural areas have higher antibiotic prescribing rates than urban areas,(5) but government interventions to optimise antibiotic use have to date focused mainly on secondary and tertiary hospitals. Published evidence reporting high prevalences of antimicrobial resistance (AMR) in China is based on national surveillance data (CARRS), point prevalence surveys and analysis of laboratory data from tertiary hospitals, but no data from settings outside urban hospitals, from rural settings or lower level (first-tier) health facilities is available. We therefore undertook an interdisciplinary mixed-method study combining epidemiological, microbiological and social science methodologies to characterise antibiotic prescribing and dispensing practices in rural health facilities and retail pharmacies. Our aims were to identify key drivers of and patient pathways to antibiotic use for common infections and to ascertain the feasibility of microbiological testing and epidemiological monitoring for ABR in rural community healthcare settings, in order to identify potential interventions to promote antibiotic stewardship and reduce the burden of antibiotic resistance.

Village clinics (outpatient only) and township health centres (which have inpatient facilities) are the lowest tier of health care in China. Patients can seek treatment at any healthcare facility but medical insurance systems incentivise use of first-tier care through greater reimbursement ratios. Around two thirds of initial consultations occur in village or community clinics, and township or community health centres receive a slightly higher proportion (17\%) than (urban) county-level hospitals (14\%). The study was conducted in Anhui Province, which has a population of 62.6 million of whom $46.7 \%$ reside in rural areas; the province's social and cultural profile is considered representative of over $80 \%$ of the Chinese population, average life expectancy is 75.08 years and per capita GDP ranks 12th among China's 23 provinces.(6)

\section{Methods}

This mixed-methods study comprised three main components: microbiological feasibility study, clinical record review, and qualitative study of antibiotic prescribing and purchasing practices. Full details of methods and analyses are provided in the published study protocol.(7) This paper synthesises findings from all study components. Data collection took place consecutively in four counties of Anhui Province, at one village clinic and one township health centre in each county. Health facilities were selected randomly from a list provided by the provincial health board of all potential facilities fulfilling set criteria (population size, location, transport links, patient footfall) in each county. Microbiological sampling and semi-structured observations were conducted in all eight health facilities, while exit surveys, interviews and observations in retail pharmacies were conducted in the first three study areas (six healthcare facilities and all local pharmacies, which varied from 4-8 in each study area).

\section{Microbiological feasibility study}

Eligible patients were recruited using consecutive sampling in each participating health facility. Inclusion criteria were $\geq 18$ years, presenting at the recruitment site for their current illness for the first time during the study period, and diagnosis of exacerbation of COPD, upper RTI with cough, sore throat or UTI. Specimen collection of sputum from RTI patients with productive cough, throat swabs from patients with sore throat and urine from patients with a UTI were performed by the attending doctor and placed into a sterilised tube for refrigeration and transportation to the participating laboratory within four hours according to a standard operating procedure. Full details of bacterial identification and susceptibility testing are provided elsewhere.(7)

\section{Semi-structured observations}

Following recruitment, participants were accompanied through their outpatient journey by a researcher who completed a recruitment proforma, clinic observation worksheet and exit survey. Presenting symptoms, tests ordered, diagnosis given and treatment prescribed were recorded through direct 
observation. At retail pharmacies, interactions between customers and pharmacy workers including symptoms presented, medicines and information requested/provided and medicines purchased were recorded over a two-week period in each shop.

\section{Structured exit surveys}

All consenting patients recruited at health care facilities completed a researcher-administered questionnaire after their consultation to elicit details of symptoms, prior treatment, views on treatment and lay diagnoses. At retail pharmacies customers purchasing antibiotics were asked to complete a similar short structured exit survey on leaving the shop.

\section{In-depth interviews}

A representative subsample of patients recruited at healthcare facilities were contacted for a follow-up interview at home, between one and two weeks after their clinic visit. A subsample of customers purchasing antibiotics over the counter were also interviewed. Semi-structured interviews were conducted with attending doctors at all participating health facilities at the end of the recruitment period and with workers at retail pharmacies.

\section{Patient record review}

After study recruitment had been completed in each of the first four participating medical facilities, researchers revisited each facility to identify the electronic records (e-records) for the observed consultations. Researchers then compared data from clinic observations and recruitment proformas with the information in the e-records, using a bespoke template to document degree of agreement. Full methodological details of this study component are provided elsewhere.(8)

\section{Patient and public involvement}

It was not feasible to involve patients or public in the design or conduct of this study as these approaches are not yet customary when undertaking health research in China; setting up a new PPI mechanism for the study was beyond our capacity. However, findings will be disseminated to participating patients and healthcare staff and patient and public consultations will be undertaken prior to the development of interventions based on the study findings.

\section{Analysis}

Questionnaire responses were double-entered into a database using EPI DATA 3.1, then extracted and analysed using SPSS and Microsoft Excel 2013. Quantitative data analysis comprised descriptive estimations and multivariate logistic regression modelling to assess factors associated with antibiotic prescription from health care professionals and test relationships between observational data and microbiological results. Initial coding of a subsample of interview transcripts in Mandarin and translated English versions was carried out by three interviewers and three UK-based researchers; codes were compared in Skype meetings to derive a dual-language coding framework for each qualitative dataset. Remaining transcripts were coded in Mandarin by interviewers using NVivo and analysed thematically. Full details of the analysis of the record review data are provided elsewhere.(8)

\section{Results}

\section{Study settings and activities}

Table 1 summarizes study sites and main research activities by site. A total of 1123 eligible patients in the clinical settings were recruited into the microbiological study and completed a structured exit interview following their consultation. The microbiological study identified 153 bacterial isolates that were tested for sensitivity to 34 commonly used antibiotics. Separately, we observed 2383 encounters in retail pharmacies and conducted exit surveys with 364 customers who had bought antibiotics. 
Table 1

Study settings and activities

\begin{tabular}{|c|c|c|c|c|}
\hline Data collection activities & Clinical & & Community & Total \\
\hline & $\begin{array}{l}\text { Township health } \\
\text { centers }\end{array}$ & $\begin{array}{l}\text { Village } \\
\text { Clinics }\end{array}$ & $\begin{array}{l}\text { Medicine } \\
\text { shops }\end{array}$ & \\
\hline Settings selected & 4 & 4 & 15 & 23 \\
\hline Encounters observed (total) & 668 & 455 & 729 & 1852 \\
\hline -symptomatic respiratory tract infections & 641 & 427 & 720 & 1788 \\
\hline -symptomatic urine tract infections & 27 & 28 & 9 & 64 \\
\hline Exit survey completed & 668 & 455 & 364 & 1487 \\
\hline In-depth interviews (total) & 49 & 30 & 24 & 103 \\
\hline -patients/customers & 37 & 24 & 16 & 77 \\
\hline -doctors/shop keepers & 12 & 6 & 8 & 26 \\
\hline Specimens collected and cultivated for bacteria & 668 & 455 & NA & 1123 \\
\hline -sputum & 440 & 243 & NA & 683 \\
\hline -throat swab & 201 & 184 & NA & 385 \\
\hline -urine & 27 & 28 & NA & 55 \\
\hline $\begin{array}{l}\text { Bacterial isolates tested for antibiotic resistance (top } 10 \text { of sputum and throat swab } \\
\text { samples) }\end{array}$ & 240 & 112 & NA & 352 \\
\hline -K. pneumonia & 63 & 22 & NA & 85 \\
\hline -H.influenzae & 44 & 13 & NA & 57 \\
\hline -H.parainfluenzae & 27 & 25 & NA & 52 \\
\hline -P.aeruginosa & 17 & 5 & NA & 22 \\
\hline -S.aureus & 14 & 4 & NA & 18 \\
\hline -H.parahaemolyticus & 10 & 7 & NA & 17 \\
\hline -M.catarrhalis & 8 & 4 & NA & 12 \\
\hline -E.cloacae & 8 & 4 & NA & 12 \\
\hline -K.aerogenes & 5 & 3 & NA & 8 \\
\hline -S.pneumoniae & 4 & 4 & NA & 8 \\
\hline
\end{tabular}

\section{Patterns of antibiotics use}

Antibiotic prescribing rates were high, with $84.3-93.2 \%$ of patients receiving a prescription containing antibiotics in the four project sites (87.8\% of RTIs and $89.1 \%$ of UTIs); of these, between $45.5 \%$ and $41.1 \%$ of prescriptions were for intravenous (IV) antibiotics. Patients presenting at township health centres were more likely to receive IV antibiotics than those attending village clinics, but their overall chance of being prescribed with any type of antibiotic was lower. Overall, $87.8 \%$ of RTI patients received antibiotic prescriptions and $35.5 \%$ of these contained two or more types of antibiotics, with the most commonly prescribed being broad spectrum antibiotics levofloxacin (31.0\% in township health centres and $30.9 \%$ in village clinics) and amoxicillin (25.6\% in township health centres and $36.3 \%$ in village clinics). $45.8 \%$ of the $1068 \mathrm{RTI}$ clinic patients reported that they had already used antibiotics in the past year, with $77.1 \%$ stating that they had obtained these from a hospital pharmacy (implying a previous clinical consultation) and $44.4 \%$ reporting that they had obtained them elsewhere. At retail pharmacies, of 2,216 observed customer purchases, 357 were observed to purchase antibiotics without prescription. Purchased medicines for RTIs at retail pharmacies contained many fewer antibiotics (16.9\%). 
Table 2

Use of antibiotics in different settings

\begin{tabular}{|c|c|c|c|c|c|c|}
\hline & \multicolumn{2}{|c|}{ Township health centres (THC) } & \multicolumn{2}{|c|}{$\begin{array}{l}\text { Village Clinics } \\
\text { (VC) }\end{array}$} & \multicolumn{2}{|c|}{ Medicine shops } \\
\hline & $\mathbf{N}$ & $\%$ & $\mathbf{N}$ & $\%$ & $\mathbf{N}$ & $\%$ \\
\hline \multicolumn{7}{|l|}{ Mode of administration } \\
\hline -Oral antibiotics & 382 & 57.2 & 251 & 55.2 & 122 & 16.9 \\
\hline -Intravenous antibiotics & 304 & 45.5 & 187 & 41.1 & 0 & 0 \\
\hline -Oral and intravenous antibiotics & 123 & 18.4 & 14 & 3.1 & 0 & 0 \\
\hline -Oral or intravenous antibiotics & 563 & 84.3 & 424 & 93.2 & 122 & 16.9 \\
\hline \multicolumn{7}{|l|}{ Number of antibiotics used in combination } \\
\hline -Zero & 105 & 15.7 & 31 & 6.8 & 592 & 82.2 \\
\hline -One & 339 & 50.8 & 236 & 51.9 & 122 & 16.9 \\
\hline -Two & 168 & 25.2 & 151 & 33.2 & 6 & 0.8 \\
\hline -Three or more & 56 & 8.4 & 37 & 8.1 & 0 & 0 \\
\hline \multicolumn{7}{|c|}{ Top 10 most commonly provided antibiotics in THC/VC/medicine shops } \\
\hline Levofloxacin/Levofloxacin/Amoxicillin & 220 & 32.9 & 158 & 34.7 & 45 & 39.1 \\
\hline Amoxicillin/Amoxicillin/Cefixime & 165 & 24.7 & 155 & 34.1 & 18 & 15.7 \\
\hline Erythromycin/Cefradine/Cefradine & 163 & 24.4 & 54 & 11.9 & 13 & 11.3 \\
\hline Cefuroxime/Ceftriaxone/Roxithromycin & 87 & 13.0 & 57 & 12.5 & 12 & 10.4 \\
\hline Ceftazidime/penicillin/Spiramycin & 41 & 6.1 & 50 & 11.0 & 10 & 8.7 \\
\hline Cefotaxime/Ampicillin/Erythromycin & 24 & 3.6 & 38 & 8.4 & 5 & 4.4 \\
\hline Piperacillin/Cefuroxime/Cefaclor & 22 & 3.3 & 20 & 4.4 & 3 & 2.6 \\
\hline Cefaclor/Roxithromycin/Levofloxacin & 21 & 3.1 & 17 & 3.7 & 3 & 2.6 \\
\hline Aloxicillin/Clindamycin/Azithromycin & 21 & 3.1 & 15 & 3.3 & 2 & 1.7 \\
\hline Cefradine/Ceftazidime/Cefalexin & 19 & 2.8 & 15 & 3.3 & 2 & 1.7 \\
\hline Other & 56 & 8.4 & 64 & 14.1 & 10 & 8.7 \\
\hline
\end{tabular}

Patients presenting to outpatient clinics with UTI symptoms were unexpectedly low $(55,4.9 \%)$ and the following statistical analysis (Table 3$)$ therefore focuses on RTI patients.

\section{Determinants of antibiotic prescribing for respiratory tract infections}

Multivariate logistic regression modelling (using IV, oral and IV/oral antibiotic use as the dependent variable respectively and the same set of independent variables as specified in Table 3) revealed that antibiotic prescription was: not linked with bacteria isolation and resistance detection; weakly linked with one type of symptom, i.e. sore throat $(\mathrm{OR}=1.64)$; strongly linked with study site $(\mathrm{OR}=4.2)$ and $>10$ days since infection onset $(\mathrm{OR}=3.33)$. Fever was found to be a predictor for prescription of intravenous antibiotics $(\mathrm{OR}=2.87)$. Overall, antibiotic prescribing was not associated with previous treatment for the current infection, though exit survey data showed that a quarter $(272,25.47 \%)$ of patients reported having used antibiotics prior to visiting the health care facility. However, patients who reported having received IV antibiotics before the study consultation had a higher chance of being prescribed IV antibiotics again and were less likely to receive oral antibiotics. Although only $8.49 \%$ and $13.42 \%$ of RTI patients had asked for IV or oral antibiotics, these clearly stated requests were strongly linked with prescription of intravenous $(O R=6.77)$ and oral $(O R=3.05)$ antibiotics, respectively.

\section{Bacterial pathogens and antibiotic resistance}

683 of RTI patients provided sputum samples, from which 71 bacterial isolates were obtained of K.pneumoniae, 52 H.influenzae, 28 H.parainfluenzae, 20 P.aeruginosa, 14 H.parahaemolyticus, 11 S.aureus, 10 M.catarrhalis 8 E.aerogenes, 8 S.pneumoniae, 6 E.cloacae and 41 other bacteria. 385 of RTI patients provided throat swab samples, from which 24 bacterial isolates were obtained of H.parainfluenzae, 14 K.pneumoniae, 7 S.aureus, 6 E.cloacae, $5 \beta$ Haemolytic Streptococcus, 5 H.influenzae, 3 H.parahaemolyticus, 2 M.catarrhalis, 2 P.aeruginosa, 2 H. haemolyticus and 13 other bacteria. 55 patients with UTI provided mid-stream urine samples which yielded 23 strains of E.coli and 5 other bacteria. 
As shown in Table 4, rates of sensitivity were higher for many bacterial species collected from sputum, swab or urine specimens when compared to the published data (CARRS, 2018). Susceptibility of E.coli from urine specimens was amoxicillin $26.7 \%$; ampicillin sulbactam $46.7 \%$; ceftriaxone $91.3 \%$; ciprofloxacin 73.9\%; gentamicin 71.4\%; cefepime 91\%; nitrofurantoin $85.7 \%$; piperacillin/tazobactam 95.6\%; and 100\% for amikacin, imipenem and meropenem - in general, higher sensitivity rates than CARRS data (Table 4). Among K.pneumoniae from respiratory tract, $>95 \%$ of isolates were sensitive to ampicillin/sulbactam, aztreonam, cefazolin, cefepime, cefotaxime/ceftriaxone, ceftazidime, cefuroxime, piperacillin/tazobactam, ciprofloxacin, amikacin, gentamicin, imipenem and meropenem, markedly more sensitive than reported through the CARRS surveillance system. In contrast to these two species, sensitivity rates in P.aeruginosa were broadly similar to CARRS. For the other species tested, H.influenzae, M.catarrhalis, S.pneumoniae or S.aureus numbers of individual strain was low or comparative CARRS data was lacking, so making comparison difficult.

Table 4

Bacterial sensitivity rates for Anhui Province from the China National Surveillance System (CARRS) and the current study(S)

\begin{tabular}{|c|c|c|c|c|c|c|c|c|c|c|c|c|c|c|}
\hline \multirow{3}{*}{ Agent } & \multicolumn{14}{|c|}{ \% susceptible } \\
\hline & \multicolumn{2}{|c|}{$\begin{array}{l}\text { K.pneumoniae } \\
(\mathrm{n}=85)\end{array}$} & \multicolumn{2}{|c|}{$\begin{array}{l}\text { H.influenzae } \\
(n=57)\end{array}$} & \multicolumn{2}{|c|}{$\begin{array}{l}\text { M.catarrhalis } \\
(n=12)\end{array}$} & \multicolumn{2}{|c|}{$\begin{array}{l}\text { S.pneumoniae } \\
(n=8)\end{array}$} & \multicolumn{2}{|c|}{$\begin{array}{l}\text { S.aureus } \\
(n=18)\end{array}$} & \multicolumn{2}{|c|}{$\begin{array}{l}\text { E.coli } \\
(n=23)\end{array}$} & \multicolumn{2}{|c|}{$\begin{array}{l}\text { P.aeruginosa } \\
(n=22)\end{array}$} \\
\hline & $S$ & CARRS & $S$ & CARRS & S & CARRS & S & CARRS & $S$ & CARRS & S & CARRS & S & CARRS \\
\hline penicillin & - & & - & & - & & 100 & 96.9 & 7.7 & 4.9 & - & & - & \\
\hline ampicillin/amoxicillin & 1.3 & - & 28.0 & 39.7 & 5.0 & - & - & & - & & 26.7 & 14.9 & - & \\
\hline ampicillin/sulbactam & 95.8 & 54.9 & 54.9 & 58.1 & 81.8 & - & - & & - & & 46.7 & 47.1 & - & \\
\hline flucloxacillin & - & & - & & - & & & & 100 & - & - & & - & \\
\hline aztreonam & 100 & 65.5 & 76.0 & - & 80 & - & - & & - & & 86.9 & 58.3 & 77.8 & 72.5 \\
\hline cefazolin & 96.2 & 43.4 & - & & - & & - & & - & & 33.3 & 21.7 & - & \\
\hline cefepime & 98.7 & 73.0 & - & & 100 & - & - & & - & & 91.3 & 76.0 & 88.9 & 85.9 \\
\hline cefotaxime/ceftriaxone & 100 & 59.3 & 100 & - & 75 & - & - & & - & & 60.9 & 40.2 & - & \\
\hline ceftazidime & 100 & 70.4 & 91.1 & - & 83.3 & - & - & & - & & 86.9 & 70.7 & 85.0 & 83.4 \\
\hline cefuroxime & 96.2 & 51.7 & 73.1 & 65 & - & & 100 & - & - & & 52.6 & 50.6 & - & \\
\hline piperacillin/tazobactam & 98.7 & 80.2 & - & & - & & - & & - & & 95.6 & 96.7 & 88.2 & 86.1 \\
\hline ciprofloxacin & 98.8 & 70.7 & 82.4 & - & 83.3 & - & - & & 94.1 & - & 73.9 & 45.3 & 80.0 & 82.0 \\
\hline moxifloxacin & - & & - & & - & & 100 & 97.2 & - & & - & & - & \\
\hline amikacin & 100 & 84.7 & - & & 100 & - & - & & - & & 100 & 96.2 & 89.5 & 93.2 \\
\hline gentamicin & 98.8 & 69.8 & - & & 91.7 & - & - & & 100 & 85.9 & 71.4 & 58.6 & 90.0 & 88.7 \\
\hline imipenem & 98.7 & 82.2 & - & & 100 & - & - & & - & & 100 & 98.2 & 84.2 & 79.9 \\
\hline meropenem & 100 & 80.0 & - & & 100 & - & 100 & - & - & & 100 & 99.1 & 94.7 & 82.9 \\
\hline tetracycline & - & & 73.7 & - & - & & - & & 81.2 & - & - & & - & \\
\hline nitrofurantoin & 48.1 & - & - & & - & & & & 100 & & 85.7 & - & - & \\
\hline chloramphenicol & - & & - & & - & & - & & - & & - & & - & \\
\hline co-trimoxazole & - & & - & & - & & - & & - & & - & & - & \\
\hline erythromycin & - & & - & & - & & 0 & 4.7 & 64.7 & 38.5 & - & & - & \\
\hline clindamycin & - & & - & & - & & 20 & 10.1 & - & & - & & - & \\
\hline linezolid & - & & - & & - & & - & - & 100 & 100 & - & & - & \\
\hline
\end{tabular}

\section{Drivers of antibiotic use}

\section{Clinical encounters}

Clinical consultations were generally short (< 5 minutes) and contained minimal exchange of information; history-taking was rare. Patients typically described their symptoms briefly and throat or pulse was sometimes examined before the doctor wrote a prescription or (in township health centres) an order for a blood test or X-ray. Consultations took place in the presence of other patients waiting their turn, returning with test results, or waiting to receive 
guidance on medication use. Most over-the-counter purchases of antibiotics at retail pharmacies also involved limited consultation, with nearly half (47\%) of medicines being decided by the customer and only $53 \%$ determined by the pharmacy worker after symptoms were described.

The brevity of encounters may explain why in exit interviews, only half $(56.2 \%)$ of clinic patients who had been observed to receive antibiotic prescriptions reported that their prescription included antibiotics, while a similar proportion of pharmacy customers (46.98\%) said the medicine they had just bought contained antibiotics (Fig. 2a). A higher proportion of clinic patients prescribed oral antibiotics confirmed that their prescriptions contained antibiotics (61\%) than those prescribed antibiotics for intravenous administration (47\%), probably because IV was described generically by doctors as a 'drip' (literally, 'hanging water').

Among the 30 doctors interviewed from all participating clinical facilities, over half indicated that diagnostic uncertainty drives antibiotic prescribing. The perceived need to provide effective treatment and ensure patient safety frequently resulted in combined prescribing (orally or intravenously) of an antibiotic and a Traditional Chinese Medicine preparation regarded as anti-viral:

"As a doctor, I think that if you aren't sure then all you can do is choose to use both types together. Because if you can't confirm what it is and if you only use, say, antibiotics (kang sheng su), anti-inflammatories (xiao yan), when in fact it is a viral infection, then you won't have good results. So all you can do is use the two together in combination" (2-1-20180131).

Although those interviewed were aware that unnecessary antibiotic use contributes to antibiotic resistance, without access to microbiological support, uncertainty regarding type of bacterial infection produced a preference for broad-spectrum and combination antibiotics:

“...if you aren't certain what type of microbial infection (jun gan ran) it is, then generally you would use broad-spectrum antibiotics (kang sheng su)... sometimes combining two or three is definitely a bit better. Why do I say a bit better? It's because you aren't certain which type of antibiotic (kang sheng su) is better for the infection" (2-1-20180201).

Such prescribing practices were described as protecting patient safety (including preventing risk of secondary infection), satisfying perceived patient demand for rapidly effective treatment, and safeguarding doctors against the potential reputational risk of failing to treat successfully.

"Now, the patients are anxious to achieve quick success and get instant benefits, if you do not use antibiotics, if the effect is not good, the patient will bring you trouble." [1-2-20170626]

Some doctors linked this concern, along with the difficulty of refusing to prescribe antibiotics, to the low social status of both rural doctors and the poor populations they serve:

“The social status of the base-level clinicians is low. [...] If the medical treatment works to [the patient's] recovery, it is your job done; but if it doesn't work, you are to be blamed.[...] Many families here are the 'left-behind' households, with young parents working as migrants away in the city [while grandparents look after the children]. Clinicians end up bearing the brunt if any social problems arise. (2-1-20180115).

Some doctors also highlighted the need to earn income for themselves and their health facility as driving their prescribing practices. Following the successful implementation of the Essential Medicines Policy (2009), health facilities cannot mark up the costs of oral medicines, but provision of clinical services including tests and parenteral drug administration are economically important: "But now the hospital in order to raise income, they give patient infusion treatment easily" [2-1-20180115]; "If you give the patient infusion, the effect is faster, it will attract more patients, the income will be better." [1-220170625]

A further driver of frequent antibiotic use is the characterisation of antibiotics as 'anti-inflammation medicine'. As described elsewhere,(9) biomedical and local understandings of infection and inflammation are elided in contemporary clinical practice in these settings. Most doctors used either the colloquial descriptor (xiaoyan yao, literally 'anti-inflammation medicine') or the specific drug name (e.g. amoxicillin) rather than the biomedical term for antibiotics in patient consultations, explaining this as necessary for communication:

“The commoners won't understand if I say 'antibiotics' (kangshengsu). They do not know 'antibiotics' (kangsheng su), they only know 'anti-inflammation medicine' (xiaoyan yao)" (2-1-20180115).

"I use the drug name of antibiotics to communicate with patient"; [...] If you say antibiotic or antibacterial drug, they can't understand" [1-2-20170625]

Interviews with patients, however, overwhelmingly confirmed that common symptoms of infection including sore throat, coughing, fever, redness and swelling are recognised as indicating the presence of inflammation. Since antibiotics are 'anti-inflammation medicine' (xiaoyan yao), patients logically regard antibiotics as appropriate treatment and frequently referred to medical experiences as a source of this knowledge:

Interviewer: 'How do you know, that when you have a cold or a cough, that you need to take anti-inflammatories?'

Interviewee: 'Everyone knows it. When you go to the drug store, drug salespersons or pharmacists will tell you this; if you go to clinics, doctors will tell you this.'

Interviewer: 'Tell you what? That you need anti-inflammatories?'

Interviewee: 'Yes. And they will treat you with anti-inflammatories.' [1-1-20170604-1]

\section{Accuracy of electronic records to monitor antibiotic prescribing}

Page 7/10 
There is considerable between-site variation in the completeness of e-records ( $0-98.7 \%$ of clinic consultations) and between physicians, as well as variability in when records are completed (from within-consultation, through days or weeks later, to annually when prescription audit requires paper records to be entered electronically). E-records are used much less frequently in village clinics than in township health centres, due to low computer literacy levels and poor connectivity. E-records were created only for individuals with health insurance. E-records were found for 781 (75.7\%) of the 1030 patients who had been observed in these clinics; the remaining $25 \%$ either did not have e-records or or were not retrievable from patient identifiers (name, date of birth). Erecord accuracy was better in relation to antibiotics (82.8\% of e-records accurately recorded what was prescribed in clinic) than for diagnosis (45.0\% accuracy) and symptoms (1.1\% accuracy) (Fig. 2b). E-records recorded antibiotic names with a high degree of accuracy (647/781, 82.8\%) and the correct doses were recorded in in 534/781 (68.4\%). E-records from township health centres $(n=636)$ recorded the administration route for antibiotics (IV/IM/oral) but not those from village clinics. Across all clinic consultations a total of 51 types of antibiotic were prescribed, while retrieved e-records documented only 38 different antibiotic types. Discrepancies were greatest for amoxicillin capsules, representing $15.6 \%$ of all antibiotic prescriptions recorded during observation but only $6.9 \%$ of antibiotic e-prescriptions; ceftriaxone sodium for injection, prescribed in $5.9 \%$ of observed consultations but $3.8 \%$ of antibiotic e-prescriptions; and levofloxacin lactate sodium for injection, which constituted $10.6 \%$ of observed antibiotic prescriptions and $8.2 \%$ of e-prescriptions.

\section{Discussion}

This study has confirmed that rates of antibiotic prescribing and consumption remain very high in rural China. Our finding that roughly a quarter of those attending low level health facilities for common RTIs or UTIs had received antibiotics for previous illness episodes in the past year, and that two-thirds of these were from a hospital pharmacy, concords with observational data on antibiotic prescribing practices in these facilities, while the findings that a further third of clinic patients had obtained antibiotics outside hospital pharmacies, and that nearly $90 \%$ of retail pharmacy customers had not previously sought treatment elsewhere, suggests that over-the-counter purchasing of antibiotics as a first resort for everyday health problems is not uncommon. Frequent antibiotic prescribing for common RTIs in clinics reinforces patient perceptions that antibiotics are appropriate for common symptoms, in turn perpetuating self-medication with antibiotics.

Analysis of patient presenting symptoms in relation to prescriptions suggests that empirical prescribing of antibiotics is particularly associated with presence of sore throat and, in the case of intravenous antibiotics, fever. Our findings further indicate that these symptoms are viewed by both patients and doctors as indicating the presence of inflammation, for which antibiotics (as 'anti-inflammation medicine') are considered appropriate. The lack of an association between presence of bacterial pathogens and prescription of antibiotics reinforces the difficulty of determining need for antibiotics in the absence of microbiological diagnostic tests. Interviews with doctors confirmed that while doctors know these conditions may not all be bacterial, they are concerned about secondary infections and, in these settings where patients have low incomes and can rarely afford time off work and domestic duties, they regard patient safety as best served by prescribing broad spectrum antibiotics, often in combination with an anti-viral traditional Chinese medicine to cover all possible causes. Suboptimal antibiotic prescribing is exacerbated by short consultations with limited history-taking and non-disclosure of prior antibiotic consumption by patients. Consequently, doctors are frequently unaware of antibiotics already taken by consulting patients, and patients are not usually told exactly what medicines they have been prescribed. This increases the potential for development of resistance to the most commonly used classes of antibiotic through repeated use (including self-medication), reflected in resistance rates of $80 \%$ to amoxicillin for E.coli and $54 \%$ for $H$.influenzae, for example. Interventions to optimise prescribing practices among rural doctors need to address patient expectations of care and, given the availability of overthe-counter antibiotics, be accompanied by measures to involve retail pharmacies in antibiotic stewardship.

Importantly, our microbiological data demonstrate that despite high rates of antibiotic prescribing and dispensing, resistance rates are low in comparison with published surveillance data. This is probably due to the presence of hospital-acquired resistant infections and referred patients who have already acquired resistance being overrepresented in the data from urban tertiary hospitals which participate in the CARRS national surveillance system.

Our study was limited to eight health facilities in rural areas of one Province and therefore may have limited generalisability for rural China as a whole, although study sites were randomly sampled within the parameters of our selection criteria. Our selection of study sites was also geographically limited by the need to transport samples to our reference laboratory in Hefei (the provincial capital) by public transportation within four hours; however, this microbiological feasibility study has nonetheless demonstrated that antibiotic resistance rates in bacterial pathogens from patients in rural China with UTI and RTI can be assessed. A further limitation of our study was very limited recruitment of UTI patients, who unexpectedly did not often present at these frontline health facilities. Reasons for this may include self-referral to more distant higher-level facilities, possibly as a consequence of stigma arising from a perceived association between UTIs and sexually transmitted infections, or low prevalence of UTI in this population, but this requires further investigation.

Our record review study has important implications for use of e-records for ABR surveillance and monitoring effectiveness of antibiotic stewardship interventions. E-records facilitate reimbursement from state medical insurance and accordingly, are created using a patient's medical insurance number; where the patient has no insurance ID, or has exceeded the individual annual reimbursement allowance for state-funded care, doctors may create e-record(s) using the ID of one or more family members to help cover costs. The use of e-records for the purpose of insurance reimbursement rather than for patient care accounts for the high degree of accuracy and completeness of antibiotic prescriptions and doses as compared with that for symptoms (1.1\%) and diagnoses (45\%). Whilst e-records are potentially a valuable source of epidemiological intelligence they should be interpreted with caution.

\section{Conclusions}

In the first study from China on antibiotic resistance in rural communities, we have demonstrated the feasibility of conducting microbiological studies to ascertain community prevalence of resistance in rural settings. Our findings show prevalence of antibiotic resistance to be consistently lower than that 
reported through national surveillance. This implies that population prevalence in China is lower than previously assumed, consistent with antibiotic resistance prevalence rates being elevated in the large urban tertiary facilities that participate in the national surveillance system.

We have also identified perception of antibiotics as anti-inflammatory, minimal doctor-patient communication, and diagnostic uncertainty as key drivers of antibiotic use. Measures to optimise antibiotic prescribing and consumption need to address health system drivers including insurance reimbursement mechanisms and socioeconomic position of rural doctors; improved diagnostic support, tailored clinical guidelines and in-service training for rural doctors to manage clinical uncertainty and patient consultations; better regulation of retail pharmacies and supply chains; and public engagement to address the perception of antibiotics as inflammation-reducing.

Comparison of electronic patient records with directly observed doctor-patient consultations shows that e-records are often incomplete and inaccurate, particularly in village health facilities. E-records' variable accuracy and completeness have important implications for monitoring and surveillance of antibiotic prescribing. Currently, research findings into the effectiveness of policies aiming to change antibiotic prescribing practices that are based on such data should be interpreted with caution.

\section{Declarations}

\section{Ethics approval and consent to participate}

The full research protocol was reviewed and approved by the Biomedical Ethics Committee of Anhui Medical University (reference number: 20170271).

Participation of patients and doctors was voluntary and written informed consent was sought from all participants.

\section{Consent for publication}

Not applicable.

\section{Availability of data and materials}

The datasets used and/or analysed during the current study are available from the corresponding author on reasonable request.

\section{Competing interests}

The authors declare that they have no competing interests.

\section{Funding}

The Newton Fund supported this study under the UK-China AMR Partnership Initiative through UK Research \& Innovation (UKRI) grant number MR/P00756/1 and National Natural Science Foundation of China (NSFC) grant number 81661138001. The funding source had no role in study design, analysis or in the decision to submit the manuscript for publication. RK, CC, MH and IO all acknowledge support from the NIHR Health Protection Research Unit in Evaluation of Interventions at the University of Bristol.

\section{Authors' contributions}

HL and DW (co-PIs) conceived the study, contributed to overall study design and led analysis of data; HL wrote the first draft with contributions from XS. XS, JChai, JCheng, RF, MC, CC and PK contributed to qualitative research design and data analysis. IO led the record review component with contributions from $\mathrm{MH}, \mathrm{RK}$ and XS on data extraction and analysis. XS also designed the project database for all study components. AMG led the microbiology component with contributions from KB and JS in development of methods for sample collection and laboratory analysis. $\mathrm{LZ}, \mathrm{XH}, \mathrm{IO}$ and $\mathrm{MH}$ developed the epidemiological design. All authors reviewed and contributed to revision of the manuscript.

\section{ACKNOWLEDGEMENTS}

The authors acknowledge the multidisciplinary study team for their help and guidance in conducting this research. In particular, we thank Tao Jiang, Xuemeng Dong, Maomao Xie and AMU graduate students for data collection, and Melissa Cole for support with coordination. This work could only be accomplished through the active involvement of many health professionals and patients in the study sites and we are very grateful for their willingness to participate in this study.

\section{References}

1. Laxminarayan R, Matsoso P, Pant S, Brower C, Røttingen J-A, Klugman K, et al. Access to effective antimicrobials: a worldwide challenge. The Lancet. 2016;387(10014):168-75. 
2. Klein EY VBT, Martinez EM, et al. . Global increase and geographic convergence in antibiotic consumption between 2000 and 2015 . Proc Natl Acad Sci USA. 2018;115(15):E3463-E70.

3. Chang Y SC, Rassamee S, et al. . Clinical Pattern of Antibiotic Overuse and Misuse in Primary Healthcare Hospitals in the Southwest of China. PLoS One. 2019;14(6):e0214779.

4. Shen X, Lu M, Feng R, Cheng J, Chai J, Xie M, et al. Web-Based Just-in-Time Information and Feedback on Antibiotic Use for Village Doctors in Rural Anhui, China: Randomized Controlled Trial. J Med Internet Res. 2018;20(2):e53.

5. Yin J LQ, Sun Q. . Antibiotic Consumption in Shandong Province, China: An Analysis of Provincial Pharmaceutical Centralized Bidding Procurement Data at Public Healthcare Institutions, 2012-16. J Antimicrob Chemother. 2018;73(3):814-20.

6. China UNDP. China national human development report 2013 - sustainable and liveable cities: toward ecological civilization. Beijing, China.: UNDP; 2013.

7. Zhao L, Kwiatkowska R, Chai J, al. e. Pathways to optimising antibiotic use in rural Anhui province, China: identifying key determinants in community and clinical settings, a mixed methods study protocol. . BMJ Open. 2019;9(8):e027819.

8. Kwiatkowska R, Shen X, Lu M, Cheng J, Hickman M, Lambert H, et al. Patients without records and records without patients: Review of patient records in primary care and implications for surveillance of antibiotic prescribing in rural China. BMC Health Services Research. 2020.

9. Lambert $\mathrm{H}$, Chen M, Cabral C. Antimicrobial resistance, inflammatory responses: a comparative analysis of pathogenicities, knowledge hybrids and the semantics of antibiotic use. Palgrave Communications. 2019;5:85.

\section{Figures}

\section{Image not available with this version}

Figure 1

Anhui Province, China

\section{Image not available with this version}

\section{Figure 2}

2a: Accuracy of patient-reported antibiotics use compared with direct observations here 2b: Accuracy of electronic records compared with direct observations here

\section{Supplementary Files}

This is a list of supplementary files associated with this preprint. Click to download.

- Table3Supplementarymaterial.docx 